Part of Journal of Research of the National Bureau of Standards, Volume 15, September 1935

\title{
ACCURACY OF MICROSCOPICAL METHODS FOR DETER- MINING REFRACTIVE INDEX BY IMMERSION
}

\author{
By Charles Proffer Saylor
}

\begin{abstract}
The accuracy of the immersion methods used in petrography for determining refractive indices is limited by the sensitivity of the criterion of match. In a series of tests, the sensitivity of three methods of ascertaining match was investigated as a function of objective aperture and size and shape of particle. The following data will illustrate the way in which experimental details, represented in the literature as equally good, can influence the accuracy of a result. A single determination of the refractive index of potassium alum has a probable error originating in the criterion of match which is as small as 0.00008 when the doublediaphragm method and an objective, $3.2 \times$, N.A. 0.11 , are employed, or as large as 0.00104 with central illumination and an objective, $45 \times$, N.A. 0.85 . The similar error with potassium chloride ranges between 0.00014 and 0.00125 , depending upon conditions. All methods are more sensitive with objectives of low numerical aperture.

The magnitude of the errors introduced by birefringence was studied by tests of a strongly birefringent compound under a broad variety of conditions. To show the influence of conditions upon accuracy, two examples may be cited. Employing a $20 \times$, N.A. 0.40 objective, plane polarized light with its vibration parallel to the horizontal crosshair, and ordinary oblique illumination from the side, the error in determining the extraordinary refractive index of mercuric cyanide is +0.0056 , but employing a $10 \times$, N.A. 0.25 objective, light with its vibration parallel to the horizontal erosshair, and double-diaphragm illumination, oblique from the side, the error is less than 0.0001 .
\end{abstract}

\section{CONTENTS}

I. Sensitivity of the criterion of match

1. Introduction $\ldots \ldots \ldots \ldots$

2. Method of central illumination _... 279

3. Method of oblique illumination $\ldots$

4. Double-diaphragm method of oblique illumination _..... 281

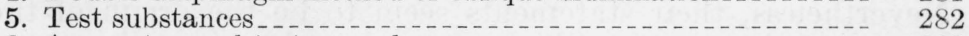

6. Apparatus and test procedure $\ldots \ldots \ldots \ldots$

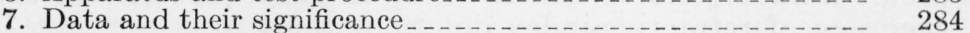

8. Influence of particle size

II. Errors originating in the birefringence of crystals

III. Conclusions _...

\section{SENSITIVITY OF THE CRITERION OF MATCH}

\section{INTRODUCTION}

Determination of refractive index by immersion is probably the most important operation in the microscopical identification of crystalline compounds. Crystals or crystal fragments are mounted in a liquid of known index. One of several criteria for indicating qualita- 
tively the relation between the indices of the crystals and the liquid is employed and the properties of the liquid are modified accordingly until, after repeated trials, a liquid is found whose refractive index matches that of the particle. In work of the highest precision, monochromatic, plane polarized light with its vibration parallel to a principal optical direction of the crystal is employed, and the efforts at matching are continued until it cannot be ascertained that the index of the crystal differs from that of the liquid.

The sensitivity of the criterion of match limits the ultimate attainable accuracy of such determinations. Other factors can, with patience, be controlled to such degree that the errors they create are small. But there is confusion and disagreement in the literature concerning the relative sensitivity of various methods of testing for a match in index. The following quotations from three of the most authoritative works in English will illustrate this situation.

Under the same conditions, the accuracy of the Becke line method and of the oblique illumination method is about the same when applied to mineral grains immersed in refractive liquids. With monochromatic illumination (strong sodium light) the refractive index of an isotropic clear mineral grain can be determined with an accuracy of \pm 0.001 , even though the diameter of the grains is only 0.01 to $0.02 \mathrm{~mm}$. *** in the Becke line method a higher power objective is advisable, the substage diafram must be closed or the condenser lowered, and practically only one mineral grain can be tested at a time. (F. E. Wright, The Methods of Petrographic-Microscopic Research, p. 96, Carnegie Inst. Washington Pub. 158, Washington, D. C., 1911.)

This bright line is the "Becke line"; it moves toward the mineral of higher index when the focus is very slightly raised. The plane of contact should be normal or nearly normal to the plane of the section for the best results. $* * *$ By the use of oblique illumination the same phenomenon may be made more distinct, so that differences of index as small as 0.001 may be detected promptly under favorable conditions. (A. N. Winchell, The Microscopic Characters of Artificial Inorganic Solid Substances or Artificial Minerals, p. 59, John Wiley and Sons, Inc., New York, 1931.)

The Becke line may be used also with the immersion method. It is more sensitive and easier to see than the light and dark borders produced by inclined illumination and at the same time it may be seen over the whole field of the microscope at once. ** * A series of tests by de Lorenzo and Riva show it to be accurate to \pm 0.001 . (Albert Johannsen, Manual of Petrographic Methods, 2d ed. p. 277, McGraw-Hill Book Co., New York, 1918.)

No one appears to have made a critical study of the actual sensitivity of immersion methods since that of $\mathrm{G}$. de Lorenzo and $\mathrm{C}$. Riva. ${ }^{1}$ They used only the method of central illumination (at least in the place indicated) and do not say what objectives were used. Nevertheless, their statements seem to be the origin of the almost occult number, 0.001 , which recurs in subsequent literature for the accuracy of refractive-index determinations.

If the identification of substances by a measurement of their refractive indices by immersion is to find its fullest usefulness in chemical analysis, the accuracy with which the refractive indices can be found should be not appreciably less than the reproducibility of the compounds themselves. It is unfortunate that the attainable accuracy should be unknown or lost in the confusion of conflicting opinions. With the hope of limiting so far as possible the uncertainty originating in the criterion of match, experiments were undertaken to learn the real sensitivity under various conditions of the method of central

${ }_{1}$ Atti Accad. Sci. Napoli [2] 10, no. 8, 9 (1901). They refer to an earlier paper by one of them in the words, "Uno di noi in una prossima nota avra occasione di indicare l'approssimazione, che con questo metodo si può ottenere, e i vantaggi che esso presenta per la determinazione dei minerali che compongono le rocce". No indications are given of where the earlier paper was published and I have not found it. 
illumination (Becke line), the method of oblique illumination, and a modification of the oblique-illumination method which, for brevity, can be called the double-diaphragm method. After preliminary experiments, each of these methods was employed in the manner which permitted it to reach the greatest sensitivity for any substance and magnification. For the highest sensitivity (and accuracy), an intense source of monochromatic light must be employed, and, if the test substance is birefringent, the light vector must vibrate parallel to a principal optical direction of the substance.

\section{METHOD OF CENTRAL ILLUMINATION}

In the method of central illumination, fragments of a material are mounted in the liquid with which its index is to be compared and viewed by a symmetrical cone of light focused, for the best results, approximately in the plane of the preparation. This point is often neglected on the erroneous assumption that a strictly parallel beam, though unattainable, is theoretically the most desirable condition. It is necessary that the angle of the cone be considerably less than the angular aperture of the objective, but the precise ratio depends upon the conditions of the particular experiment. When the objective is elevated from the position of truest focus, a bright line or zone appears and moves from the interface between the material and the immersion fluid toward the medium of higher index. If the objective is lowered, the reverse effect occurs. When, for light of a single wave length, the index of the liquid is made to match that of the material, the bright line disappears and the outline of the fragment vanishes.

Among other things, the sensitivity of the method depends upon the shape and size of the fragment, the angle of the cone of light, its luminous intensity, and the numerical aperture ${ }^{2}$ of the objective.

\section{METHOD OF OBLIQUE ILLUMINATION}

When viewed by oblique illumination, a fragment mounted in a liquid of lower index appears (by inversion in the microscope) to be bright on the side from which the light actually comes, and shaded on the opposite side. If the fragment is mounted in a liquid of higher index, the conditions are reversed. At least nine methods for obtaining oblique illumination have been described, seven being listed by Wright. ${ }^{3}$ The best, in the most perfect form that is ever really used, is as follows. The light source, or a condensing lens placed before it, is sharply focused by the condenser in the plane of the preparation. The iris diaphragm of the condenser is so adjusted that the condenser aperture is a little greater than that of the objective. The ocular is removed or the Bertrand lens is focused upon the back element of the objective. An opaque stop, the end of a celluloid ruler, for example, is inserted in the condenser system at such a level that a sharp image of it is formed at the back surface of the objective. It is pushed across until about a fifth of the diameter of the back surface of the objective is still bright. Then, if the Bertrand lens is removed or the ocular replaced,

2 Concerning numerical aperture it should be noted that in each of the places cited a high numerical aperture or, what is equivalent to it, a high-power objective, has been recommended for Becke line effects as yielding greater sensitivity, but that such conclusions are not confirmed by the experiments here reported (see figs. 5, 6, 7, 8, 9, and 10).

F. E. Wright, Am. J. Sci. 185, 63 (1913). 
the entire field of the microscope is seen to be dimly but uniformly lighted. The objective mounting, by serving as a second diaphragm, intercepts about half of the light from the stage. Since the opaque

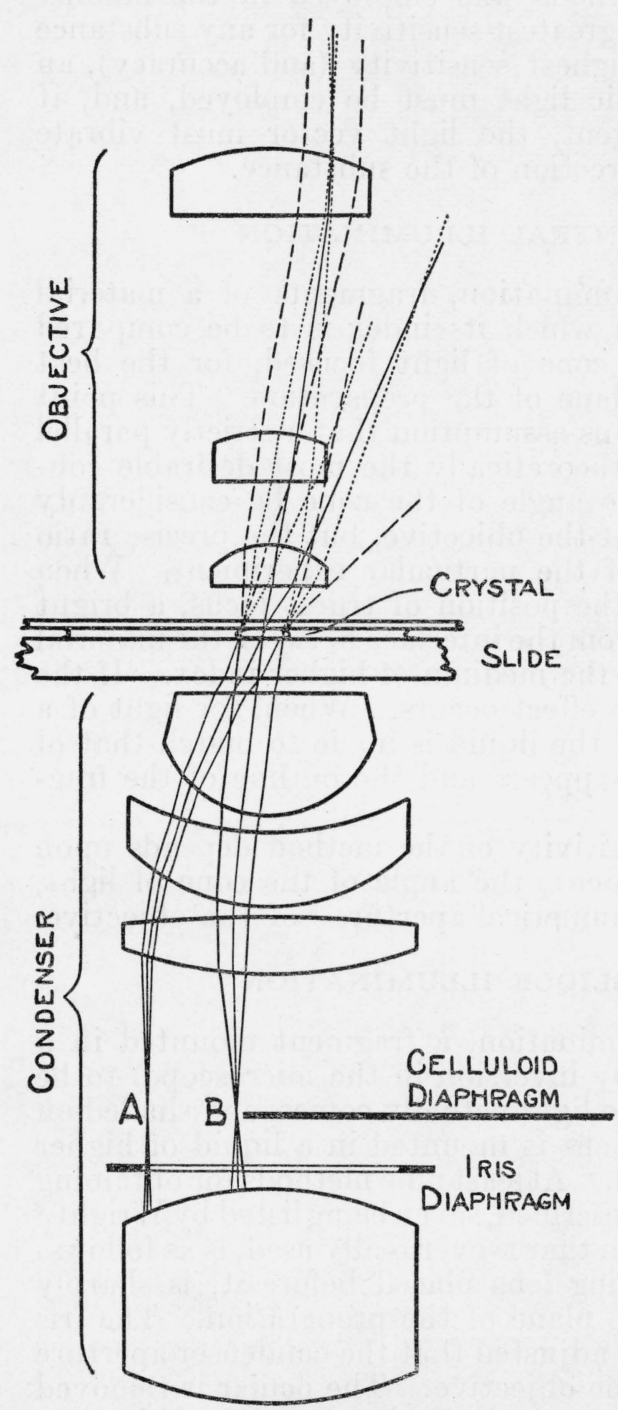

Figure 1.-Oblique illumination.

Condenser, diaphragms, preparation, objective, and ray paths. The rays, drawn as full lines before they reach the preparation, are refracted if they strike the edges of the crystal. The courses they then take are drawn as dashed lines and the courses they would have taken if not deflected are shown as dotted lines. Some rays, striking such parts of the preparation that they are not appreciably deflected. have been continued through as full lines. The index of the crystal is assumed lower than that of the liquid. above, in which it is at such
a level that no part of the field is darkened more than any other. Its advantage is that all light reaching any part of the field comes along stop in the condenser has been made approximately conjugate with the objective mounting, both the rays which are intercepted and those which pass through come uniformly from all parts of the field. Optical inhomogeneities in the field change the path of the rays, and relatively small angular deviations may cause them to fall on a different side of the boundary imposed by the objective mounting. From the chosen point $A$, figure 1, rays diverge and spread to every part of the field, but unless their paths are changed by the preparation all are stopped by some part of the objective mounting. If the index of the liquid is greater than that of the crystal, the ray which strikes the left side of the crystal in figure 1, is deflected to the left and caused to fall within the aperture of the objective. Thus the left side of the crystal (apparently the right side) is made brighter. Rays from point $B$, on the other hand, pass into the objective and serve to illuminate all parts of the field unless, as by striking the right edge of the crystal, they are so deflected as to be intercepted by the objective mounting. In such manner originate the bright and shaded portions of particles immersed in a fluid of different index.

Both theoretically and practically, the best setting of the opaque stop in the condenser is the one described 
a chosen path. With the diaphragm at any other level, the oblique light reaching any part of the preparation is always mixed with light from other directions, with an uncertain amount of directly transmitted light, and even with light coming obliquely from the opposite direction. This tends to obscure the evidences of heterogeneity.

The sensitivity of the method is influenced by the size and shape of particles, the numerical aperture of the objective, the brightness of the light, the diaphragm settings, and by the perfection of corrections in the marginal zones of the objective, since these alone serve for the formation of images.

\section{DOUBLE-DIAPHRAGM METHOD OF OBLIQUE ILLUMINATION}

In section 3 the ordinary method of oblique illumination was explained by considering the mounting of the objective as a second diaphragm which intercepts most of the undeviated light from the condenser. It is a logical extension from this to increase the sensitivity by placing another diaphragm at the objective in a position which is deliberately chosen to give the best results. Wright ${ }^{4}$ described a method in which two diaphragms were used, but since one was placed below the polarizer and the other between the objective and the slide, they formed diffuse images in the field of vision. Only a band at the center could be successfully employed and the method appears to have fallen into disuse.

The double-diaphragm method which has been tested is a modification of the foregoing. A piece is cut from a thin filing card in the form:

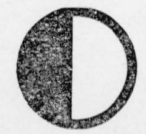

This is laid upon the back element of the objective so as to block the right half of the objective aperture. The edge of a piece of sheet aluminum or the end of a celluloid ruler is pushed into the condenser system from the right. Its image at the back of the ohjective appears from the left. It is adjusted vertically so as to form an image in the plane of the cardboard piece resting on the back element of the objective. When the entire field of the microscope is dimly but uniformly lighted, the diaphragm in the condenser is clamped in position. By removing the ocular, the back of the objective is seen as follows:

From the chosen point A, figure 2, rays spread to every part of the field, but unless their paths are changed by the preparation all are intercepted by the special diaphragm in the objective. The crystal, however, has a different index of refraction than the fluid and that ray which strikes it on its left edge is deflected and caused to fall within the unobscured portion of the objective aperture. Rays from the point $\mathrm{B}$, on the other hand, passing through the objective serve to illuminate all parts of the field unless portions of the crystal - the right edge - deflect them so that they are stopped by the special diaphragm. When a test is being performed and a particle has been made nearly invisible, the iris diaphragm and the mask in the condenser are adjusted to such positions that the light and dark shadings

\footnotetext{
4 J. Wash. Acad. Sei. 4, 389 (1914).
} 
of the particle have a maximum distinctness. This setting does not as a rule need to be changed so long as the same objective is employed.

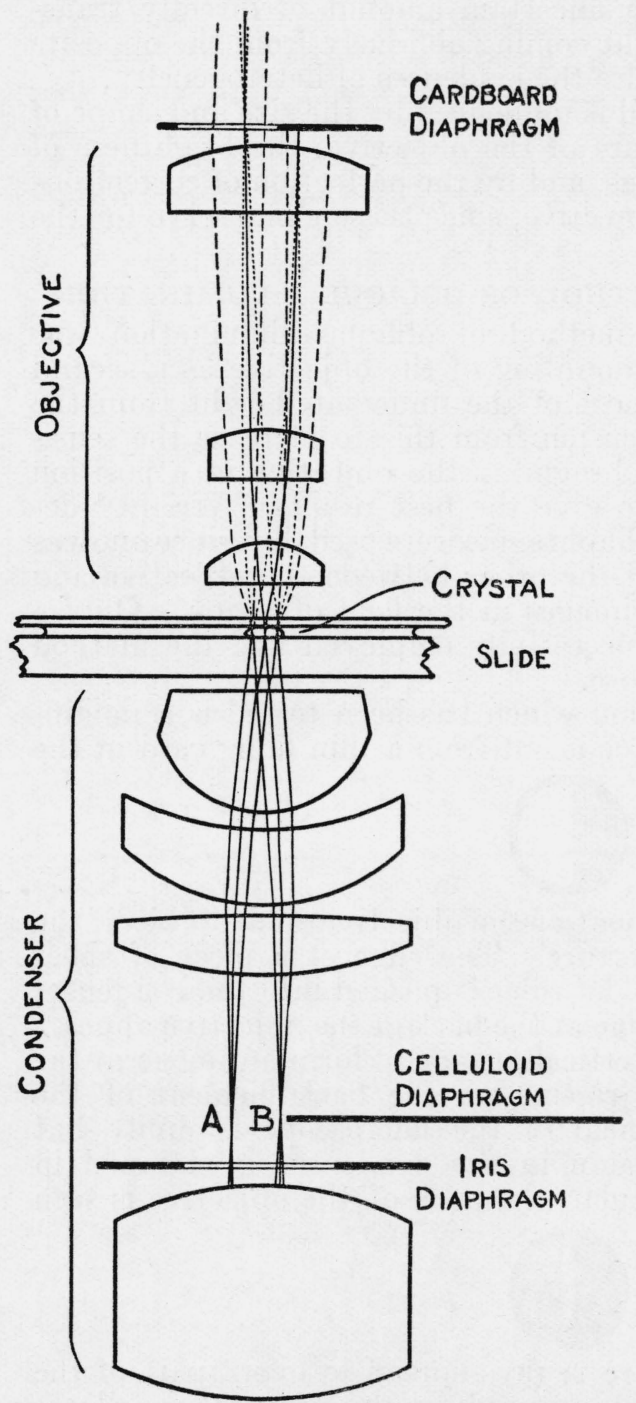

Figure 2.-Double-diaphragm method of oblique illumination.

Condenser, preparation, objective, iris diaphragm, special diaphragms in condenser and objective, and ray paths. The rays, drawn as full lines before they reach the preparation, are refracted if they strike the edges of the crystal The courses they then take are drawn as dashed lines and the courses they would have taken if not deflected are shown as dotted lines. Some rays, striking such parts of the preparation that they are not appreciably deflected, have been continued through as full lines. The index of the crystal is lower than that of the liquid.

ilar to those of spheres. During tests, all substances but the crushed glass were so oriented that refracting surfaces were normal to the plane of incidence.
The double-diaphragm method of oblique illumination has been tried, in the form described above, with five types of petrographic microscopes that were accessible to the author. There were differences in ease of manipulation, but a critical placing of the two diaphragms was possible with all of them.

The sensitivity of this method also is dependent, among other things, upon numerical aperture, size and shape of particle, correctness of diaphragm settings, and brightness of light.

\section{TEST SUBSTANCES}

The sensitivity of these methods was tested practically by using four substances, selected because of their shapes and the values of their refractive indices. Potassium chloride grown directly upon the slide has no inclined faces, the vertical interfaces between crystal and liquid being the only ones by which failure of crystal and liquid to match can be observed. Potassium alum, grown upon the slide, has no vertical surfaces, and the octahedron faces inclined $19^{\circ} 28^{\prime} 26^{\prime \prime}$ from vertical provide all useful refraction effects. Particles of crushed borosilicate laboratory glass have irregular surfaces and resemble in configuration the powders on which petrographic determinations are usually made. Glass wool was chosen because it has no angular edges and because its refraction effects are sim- 


\section{APPARATUS AND TEST PROCEDURE}

The general scheme of the tests was that of observing one particle immersed between slide and cover glass in a known liquid and then, using each of the three methods for determining match, the conditions were so modified that the index of the particle repeatedly became just detectably higher and then just detectably lower than the index of the liquid. To avoid spurious effects, a setting was considered for recording only when two opposite sides of the particle were faintly visible and indicated the same relation between the indices of the particle, and the immersion liquid.

Gaubert's ${ }^{5}$ plan of varying the temperature to adjust the index of an immersion liquid with respect to a particle was adopted. Since the temperature coefficient of refractive index is from 3 to 1,000 times as great for liquids as for crystals having about the same index, such adjustments are usually possible with a liquid of suitable index. In the present study, mounts were made upon a special slide or cell, ${ }^{6}$ with which temperature control is secured by running a rapid stream of water between microscope cover glasses. This device, which has about the same optical thickness as an ordinary microscope slide, allows the entire illuminating cone of the condenser to be employed when desired and permits normal relationships between condenser, preparation, and objective. In consequence, the apparatus for controlling temperature imposed no modification upon any method of determining match. The temperature of the water circulating through the slide was regulated by means of a valve which adjusted the proportions of hot to cold water. The hot and cold water were drawn from overflow tanks, suspended from the wall, through which the ordinary tap water of the building was circulated. Thus by the shifting of a single calibrated lever, the temperature of a liquid in which particles were mounted could be precisely regulated between 15 and $50^{\circ} \mathrm{C}$. One-tenth of a degree corresponded to about $0.7 \mathrm{~mm}$ on the graduated dial.

The immersion liquids were chosen, so far as possible, from a series of complex esters ${ }^{7}$ of extraordinary stability. Potassium chloride crystals were mounted in bisethoxyethyleneglycol phthalate. For potassium alum and the crushed borosilicate glass, appropriate mixtures of bisethoxydiethyleneglycol adipate and bisbutoxyethyleneglycol phthalate were employed. These liquids were thoroughly stable and did not suffer change in index, so far as could be detected, after several days at temperatures fluctuating between 25 and $40^{\circ} \mathrm{C}$. For glass wool, no fully satisfactory liquid seemed to be available. The mixture of mineral oil and monochloronaphthalene which the writer uses as an index liquid of 1.520 was employed, but by the loss of the monochloronaphthalene its index slowly became lower during use. The results with the glass wool are given, therefore, not in terms of relative refractive index, but as deviations from a mean value (see figure 6). For a series of temperatures between 20 and $40^{\circ} \mathrm{C}$, the index of each liquid was determined upon a special, low-reading, Abbe refractometer ${ }^{8}$ with which, by applying a correction, it is possible to obtain results accurate within 2 or 3 units in the fifth decimal place.

\footnotetext{
${ }^{5}$ Bul. soc. franç. minéral, 45, 89 (1923).

6 C. P. Saylor, J. Research NBS 15, 97 (1935) RP814

7 These compounds were synthesized by C. H. Binkley, to whom the writer is much indebted.

8 Warren P. Valentine, Philadelphia, Abbe refractometer 368.
} 
In all tests, temperatures were determined by means of a 5 -junction, copper-constantan thermocouple, with its warm end placed between the slide and cover glass so that it was just at the edge of the field when the $45 \times$ objective was used. The thermocouple wire was no. 38 AWG. Junctions were electrically welded and filed at the welds to remove all excess metal. They were arranged so that the tips were in one straight line and the wires all in the same plane. They were held in this position by an insulator $1 \mathrm{~cm}$ back from the tips so that it was well outside the cover glass. The thermocouple caused a separation of about $0.1 \mathrm{~mm}$ between slide and cover glass. A bath of shaved manufactured ice and previously chilled distilled water in a vacuum flask served to maintain the cold junction. All readings were made with the same potentiometer as had been employed in calibrating the thermocouple itself.

Four different objectives were used: no. $1,3.2 \times$, N.A. 0.11 ; no. 3 , $10 \times$, N.A. 0.25 ; no. $4,20 \times$, N.A. 0.45 ; and no. $6,45 \times$, N.A. 0.85 . The same $10 \times$ ocular was used throughout. All tests were made with the filtered 577 and $579 \mathrm{~m} \mu$ lines of the mercury vapor arc. The laboratory was not darkened during any of the tests although the microscope stage was shaded whenever desirable.

The most critical point was that of determining the temperature corresponding to the least detectable difference in index between a particle and the immersion liquid. When a temperature was found at which the index difference was small but still readily perceptible, the conditions were adjusted in every detail to make the evidences of failure to match as conspicuous as possible. This setting, once made for a given preparation, objective, and method, was maintained for all tests. It was checked, however, at the completion of a run after the attainable sensitivity was known. Then the temperature was adjusted upward and downward to obtain a series of readings. A temperature setting was made which was lower than the conditions producing invisibility. As this temperature was slowly raised, the contrast between the particle and the liquid diminished. When the least perceptible gradations on opposite sides of the particle showed that its index was lower than that of the liquid, the potentiometer was read, but to guard against changes while the potentiometer was being set the appearance of the field was checked before making a record. A similar reading was made for conditions where the index of the particle was higher than that of the liquid. Thus a series of readings was obtained for each objective, substance, and method, there being as a rule five readings when the index of the liquid was above and five when it was below that of the chosen grain.

\section{DATA AND THEIR SIGNIFICANCE}

Figure 3 for potassium chloride, figure 4 for alum, figure 5 for crushed glass, and figure 6 for glass wool give the results of tests. Opposite the designation of each objective are plotted all the index determinations made with it. The determinations by each method are arranged in columns. A plus sign indicates that the index of the liquid can be seen to be higher than that of the particle, and a minus sign that the index of the liquid is lower than that of the particle. In each section, the points are arranged from left to right in the order of observation. The vertical distance between the rows 
of plus and minus signs is a measure of the uncertainty of any single determination. From the data several conclusions are possible.

(a) Under similar conditions, the double-diaphragm method is more sensitive than either of the others.

(b) The ordinary method of oblique illumination is usually the least sensitive. In two cases out of ten the method of oblique illumination

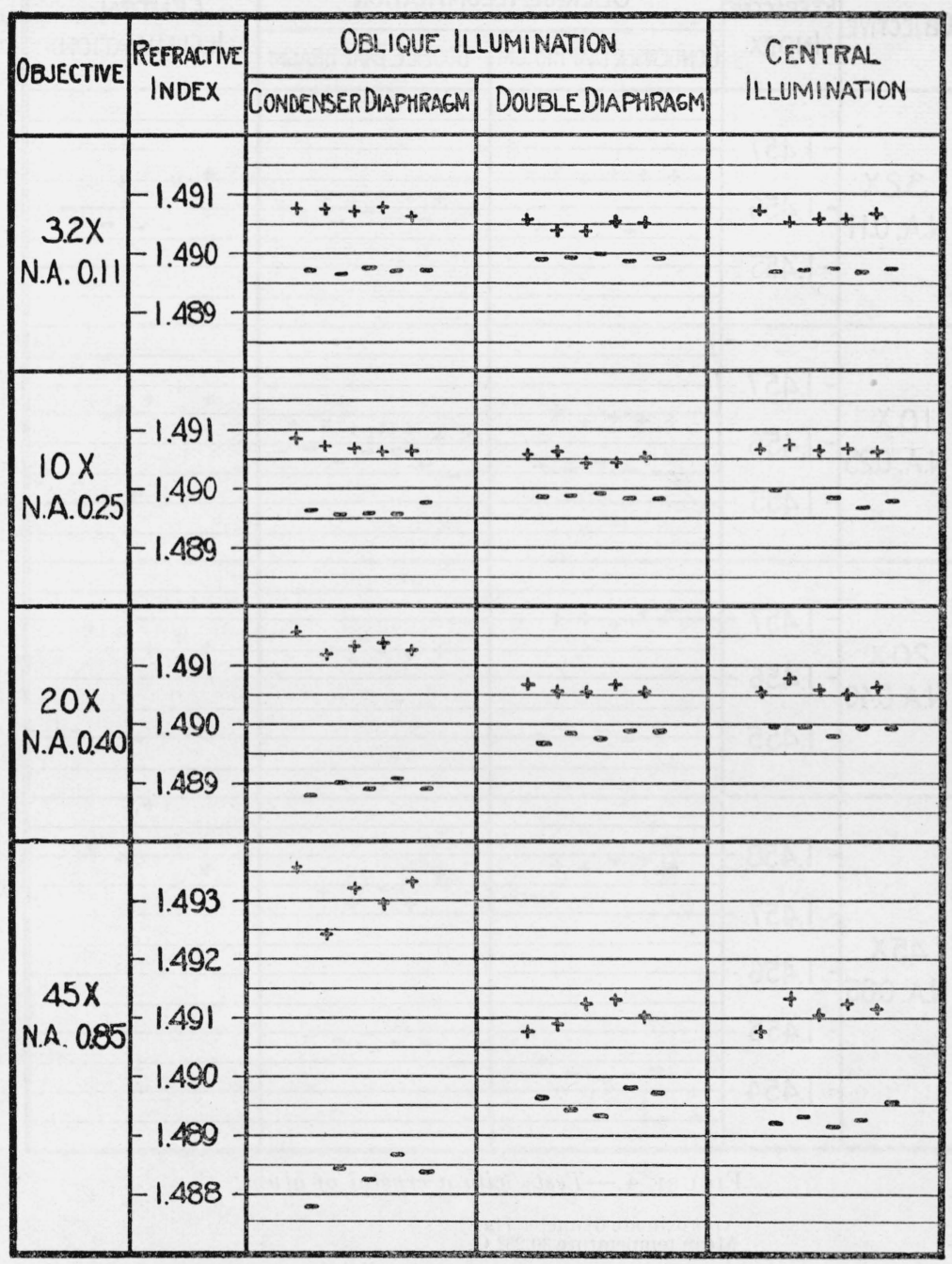

FIGURE 3.-Tests with a crystal of potassium chloride.

Approximate diameter $65 \mu$.

From International Critical Tables, $n_{578}^{38.88}=1.49034$

seems to be slightly more sensitive than the method of central illumination, but the difference is about the same as the uncertainty of the tests.

(c) By all methods, low-power objectives give greater sensitivity than high-power objectives. For the method of central illumination, 
this is unsupported by most of the literature, ${ }^{9}$ but it is a theoretical necessity. The immersion methods are all deviation methods. In general, a particle immersed in a liquid deflects a certain part of the light rays which strike it, but as the difference in index between a particle and its environment becomes progressively smaller, the

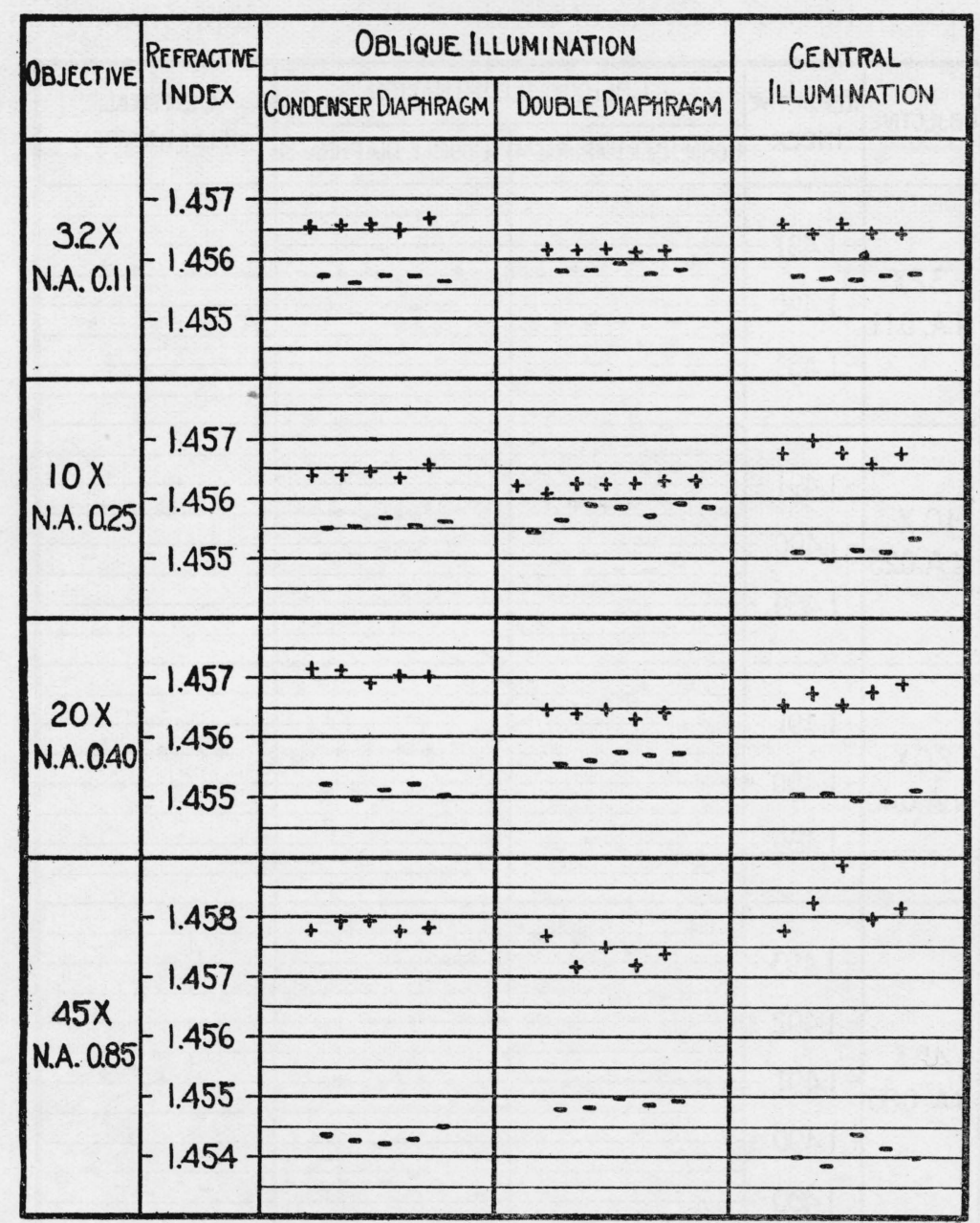

FIgURE 4.-Tests with a crystal of alum.

Approximate diameter $74 \mu$.

Mean temperature $30.25^{\circ} \mathrm{C}$.

From International Critical Tables, $n_{\mathbf{8 7}}^{30.25}=1.45618$.

extreme angular deviation decreases until the effect can no longer be detected. A given angular deviation is a smaller part of the angular aperture of a high-power objective than of a low-power one, hence a small difference in index with a low-power objective is equivalent to a larger difference with an objective of higher power.

E. M. Chamot and C. W. Mason, Handbook of Chemical Microscopy, vol. I, p. 369, John Wiley and Sons, New York, 1930, correctly recommend an objective of low aperture. 
(d) The method of central illumination is subject to relatively large differences, depending upon the shape of a particle. The sensitivity is about twice as great with a substance like potassium chloride (vertical surfaces) as with alum (inclined faces). Unless the surface lies within the cone of illumination so that light can approach from both sides, there is a great loss of sensitivity. In examining a powdered preparation in which the orientation of the surfaces is heterogeneous, an observer sensibly chooses those parts of fragments which

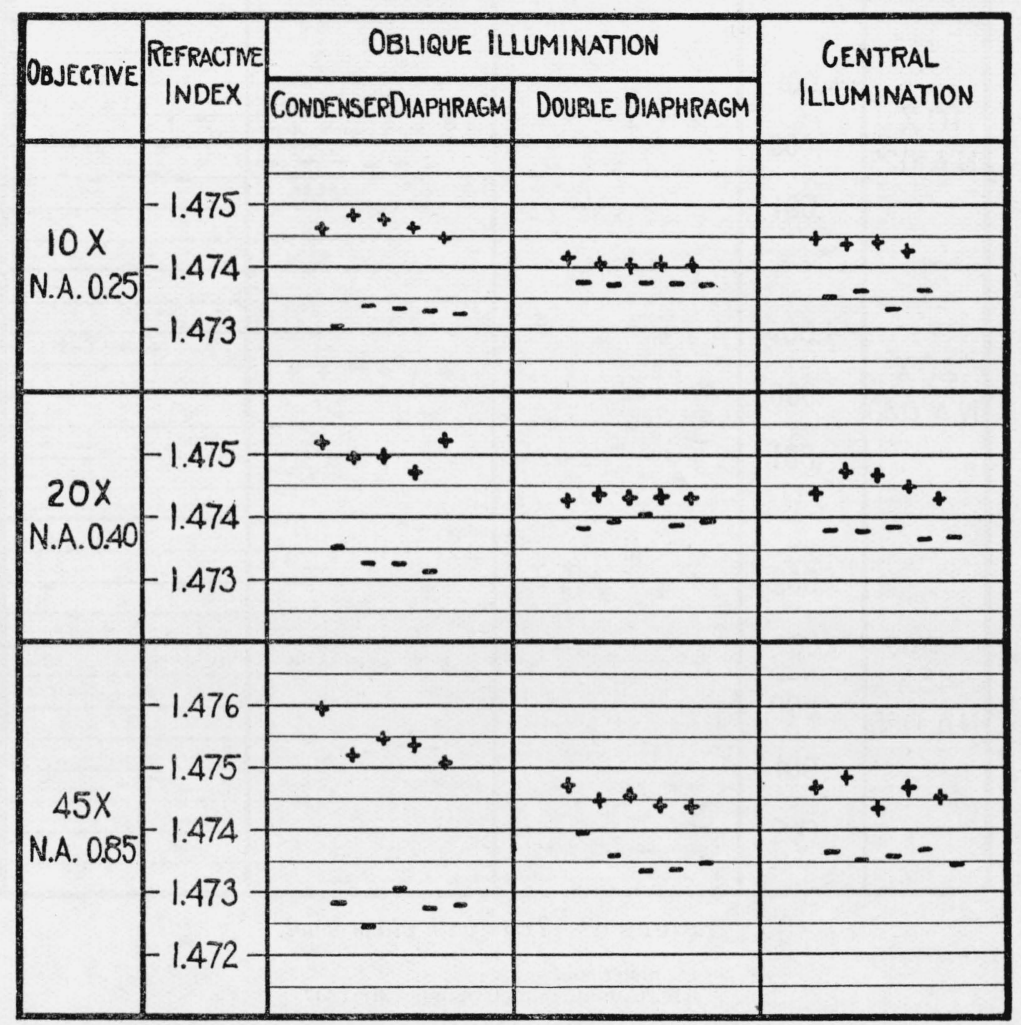

FigURe 5.-Tests with crushed borosilicate laboratory glass.

Particles roughly equidimensional, diameter approximately $85 \mu$.

show the most distinct phenomena. For such a reason, the results reported in this paper for crushed glass by the method of central illumination showed about the same sensitivity as did the results for potassium chloride. The explanation of Becke is the true one, and statements like that in a book review by Tunell and Morey ${ }^{10}$ are founded upon an error concerning the origin of phenomena really observed in practical petrographic identifications.

$10 \mathrm{~J}$. Am. Chem. Soc. 54, 2575 (1932). They write, "The phenomena observed under the microscope with central illumination of powdered grains immersed in a liquid, sometimes called the 'Becke' line, are improperly so-called; the expression, 'Becke line' was coined by Salomon to designate the phenomena associated with total reflection at a vertical interface, which differ in theoretical interpretation from the phenomena obtained with central illumination of irregular grains; the latter are properly known as the central illumination effect." 
(e) Neither method of oblique illumination exhibits so much dependence upon particle shape as the Becke line method.

The data presented in figures $3,4,5$, and 6 represent conditions of failure to match. Visible optical phenomena indicate that the index of the immersion fluid lies either above or below that of the

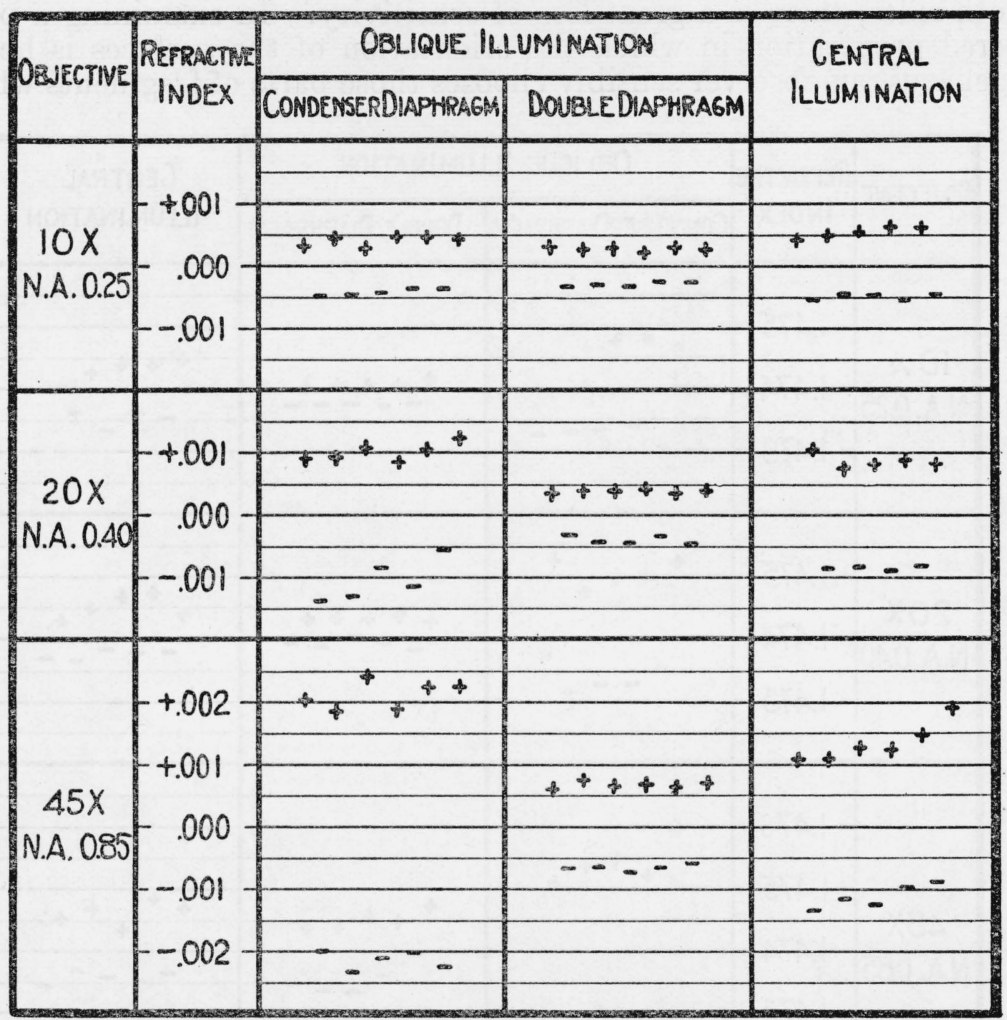

Figure 6.-Tests with glass wool.

Diameter $30 \mu$.

Approximate refractive index, $n=1.517$.

material studied. In a practical determination of refractive index by the common procedure of changing liquids until one is found in which the immersed particle is invisible, the probable error at match would be approximately one-fourth ${ }^{11}$ of the difference between the upper and lower rows of points. On this basis table 1 has been formed. It lists the probable errors of single determinations by matching of substances shaped like alum and potassium chloride.

\footnotetext{
11 The usual probability relations do not apply in this case. When a particle is being matched, the properties of the liquid are not ordinarily varied continuously. Instead, the particle is compared with previously prepared mixtures. After invisibility has been secured there is no greater likelihood that the true index lies in one part than another of the zone of uncertainty.
} 
TABLE 1.-Probable error of a single determination resulting from uncertainty of match

\begin{tabular}{|c|c|c|c|c|}
\hline \multirow{2}{*}{ Objective } & \multirow{2}{*}{ Substance } & \multicolumn{2}{|c|}{ Oblique illumination } & \multirow{2}{*}{$\begin{array}{l}\text { Central } \\
\text { illumina- } \\
\text { tion }\end{array}$} \\
\hline & & $\begin{array}{l}\text { Single dia- } \\
\text { phragm }\end{array}$ & $\begin{array}{c}\text { Double } \\
\text { diaphragm }\end{array}$ & \\
\hline $3.2 \times$ & $\left\{\begin{array}{l}\text { Alum } \\
\mathrm{KCl}\end{array}\right.$ & $\begin{array}{r}0.00023 \\
.00026\end{array}$ & $\begin{array}{r}0.00008 \\
.00014\end{array}$ & $\begin{array}{r}0.00020 \\
.00023\end{array}$ \\
\hline $10 \times$ & $\left\{\begin{array}{l}\text { Alum }_{\ldots} \\
\mathrm{KCl}\end{array}\right.$ & $\begin{array}{l}.00021 \\
.00027\end{array}$ & $\begin{array}{l}.00011 \\
.00016\end{array}$ & $\begin{array}{l}.00041 \\
.00022\end{array}$ \\
\hline $20 \times$ & $\left\{\begin{array}{l}\text { Alum } \\
\mathrm{KCl}_{-}-\end{array}\right.$ & $\begin{array}{l}.00049 \\
.00048\end{array}$ & $\begin{array}{l}.00019 \\
.00020\end{array}$ & $\begin{array}{l}.00042 \\
.00017\end{array}$ \\
\hline $45 \times$ & $\left\{\begin{array}{l}\text { Alum } \\
\mathrm{KCl}\end{array}\right.$ & $\begin{array}{l}.00088 \\
.00125\end{array}$ & $\begin{array}{l}.00062 \\
.00037\end{array}$ & $\begin{array}{l}.00104 \\
.00045\end{array}$ \\
\hline
\end{tabular}

As a consequence of the conditions which are chosen for estimating match, the probable error in determining the refractive index of a crystal may be as small as \pm 0.0001 or as large as \pm 0.0010 .

\section{INFLUENCE OF PARTICLE SIZE}

A few tests have been performed to learn the effect of the size of particles upon the sensitivity of refractive-index determinations. Since the results qualitatively verified deductions from the data of table 1 , and since any complete investigation would be most involved and fatiguing, the findings have been allowed to remain in a somewhat fragmentary state.

Spheres of glass, ${ }^{12}$ approximately $2 \mu$ in diameter, were examined by the double-diaphragm method with the 10 and $45 \times$ objectives. With the $10 \times$ objective, the spheres were not visible if the refractive index of the liquid was between 1.4711 and 1.4733 . With the $45 \times$ objective, the limits were 1.4676 and 1.4745 . In table 2 the probable error of a single determination deduced from these data is compared with the probable error with the glass wool (diameter $30 \mu$ ).

TABLE 2.-Influence of size upon the probable error of a single determination

\begin{tabular}{|c|c|c|}
\hline Objective & $\begin{array}{c}\text { Glass wool } \\
30 \mu \\
\text { diameter }\end{array}$ & $\begin{array}{c}\text { Glass } \\
\text { spheres } \\
2 \mu\end{array}$ \\
\cline { 1 - 2 } $10 \times$ & 0.00014 & 0.0006 \\
$45 \times$ & .0017 \\
\hline
\end{tabular}

By using particles one-fifteenth the diameter, the probable error has increased approximately fivefold. Obviously this rate of change must increase more rapidly as the limit of resolution is more nearly approached and thus the probable error become so large as to lose all meaning when the particles are irresolvable.

\footnotetext{
12 These spheres of borosilicate laboratory glass were made by Dana L. Bishop, who has described their preparation. BS J. Research 12, 177 (1934) RP642.
} 
Determinations of refractive index by immersion are always made under bad conditions of resolution-the iris diaphragm is nearly closed, a stop in the condenser allows only the most oblique light to enter, or a diaphragm in the objective excludes most of the light which has not already been stopped by a diaphragm in the condenser. Down to a point where it becomes difficult to make out forms, the size of a particle does not have much influence, but for particles smaller than this the sensitivity falls off rapidly. Then, it is only by relaxing some of the conditions imperative for high sensitivity that resolution itself can be secured. Thus it is that those methods which are most effective with large particles permit the greatest sensitivity with particles near the limit of resolution.

\section{ERRORS ORIGINATING IN THE BIREFRINGENCE OF CRYSTALS}

In determining refractive indices by immersion methods, petrographers usually assume that the light vibrates normally to the axis of the microscope. They assume, in other words, that under all circumstances the beam of light is so little oblique that negligible errors are introduced by disregarding its inclination. Obviously there are no errors when, as in section I the substances investigated are isotropic. Just as obviously, the errors will be negligibly small when the birefringence is sufficiently slight. But substances of strong birefringence make up a large proportion of the cases in which the values of fundamental optical constants have been disputed.

Under a number of conditions it is possible to calculate ${ }^{13}$ sufficiently well the errors which will result from assuming the light to be axial when, in reality, it is a composite of rays traveling many paths of greater or less inclination. The actual determination is a subjective integration of the information supplied by all these rays. When the difference between their errors is small as compared with the average error, it is satisfactory to simplify the mathematics and compute an average value. Otherwise the information may be gleaned more satisfactorily by a series of experiments. To accomplish this the errors generated by birefringence were studied on an actual crystal as functions of the numerical aperture of the objective, the criterion of match, and the relation between the plane of obliquity and the direction in the crystal corresponding to the refractive index being determined.

Mercuric cyanide crystallizes in the tetragonal system. When grown on the microscope slide it almost invariably rests upon a prism face. End forms are reasonably numerous, there being first- and second-order pyramids and possibly also sphenoids and pinacoids, but their descriptions are not pertinent to this investigation. Mercuric cyanide is strongly birefringent and uniaxial negative. The value of $\epsilon$ falls in a region served by one of the most satisfactory of immersion liquids, dibutyl phthalate. Since the birefringence of the compound is so extreme, $\omega-\epsilon=0.1563$, and since the limiting value $\epsilon$ can be determined only by using rays which travel in the basal plane of the crystal (which is perpendicular to the stage of the microscope),

${ }^{13}$ In a theoretical discussion, F. E. Wright, J. Wash. Acad. Sci. 5, 105 (1915), calculated the errors which should be caused by birefringence when the method of central illumination and the ordinary method of oblique illumination are employed. In the main, the effects which he calculated are consistent with those found experimentally in this study, but divergences with high-power objectives are of such magnitude that they ought not be neglected. 
any deviation from this plane has a relatively large effect upon the apparent value of $\epsilon$. Mercuric cyanide proved, therefore, to be almost ideally suited for these tests. With other substances, it is to

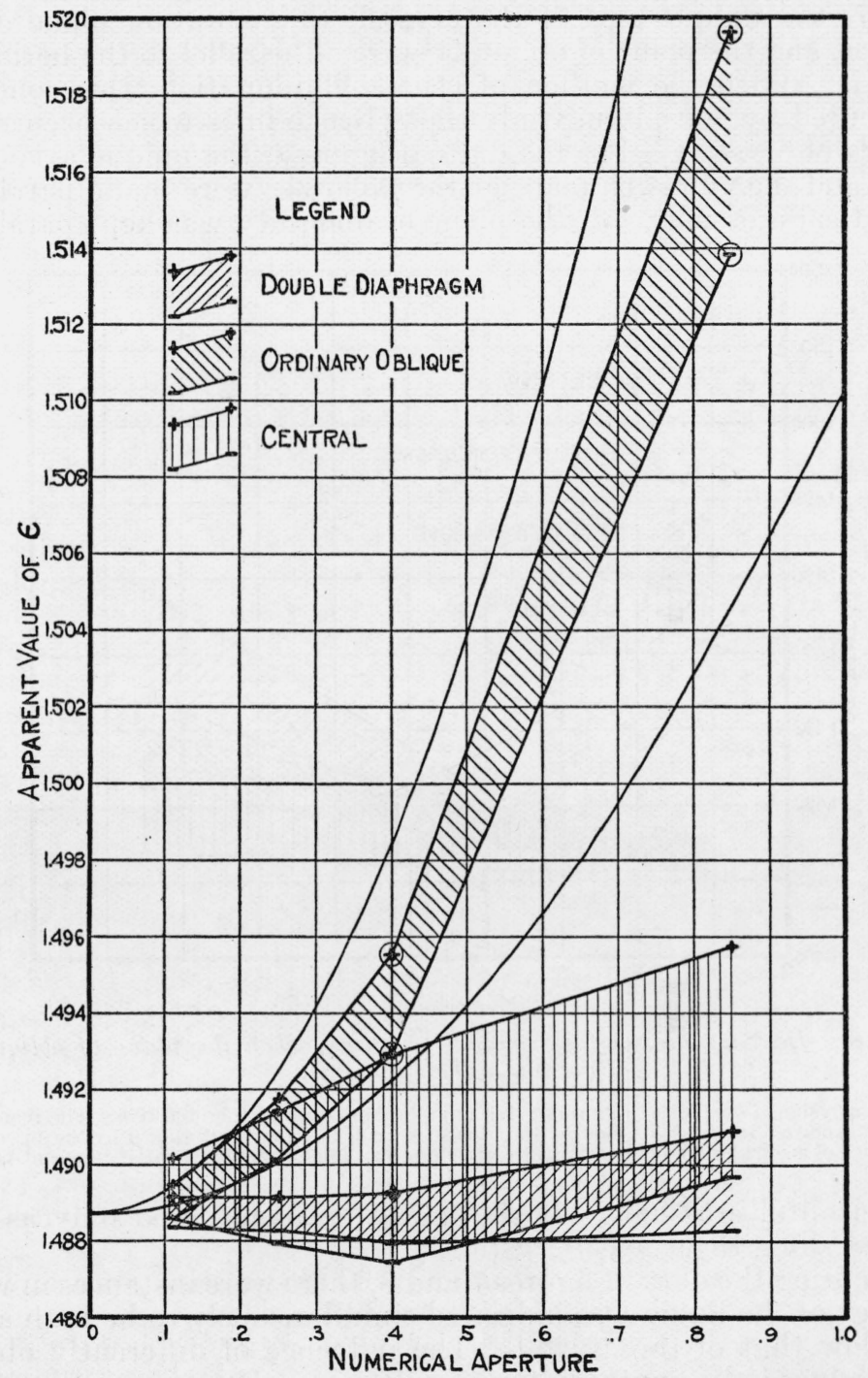

FiguRE 7.-Determination of $\epsilon$, mercuric cyanide, when the plane of obliquity contains the optic axis.

The apparent value of $\epsilon$ is plotted against the full numerical aperture of the objective. The inaccuracies that are introdued into average of a zone lies above 1.4887 (true $\epsilon$ as determined from the data of this figure and figure 8). Calculated values for the ordinary method of oblique illumination are plotted as smooth curves.

be expected that errors will differ approximately in proportion to the birefringences.

Following the general procedure of section I, $\epsilon$ of mercuric cyanide was bracketed by the three methods of matching, and the same four objec- 
tives, as well as for two orientations of the plane of obliquity with respect to the vibration direction being investigated. The data are presented in figures 7 and 8 . To secure the results represented in figure 7 , the unique axis of the crystal, the vibration plane of the polarizer, and the plane of obliquity were all parallel to the horizontal crosshair. With the method of central illumination, this condition was secured by considering only those Becke lines which occurred at the ends of crystals. For the data of figure 8, the unique axis of the crystal and the vibration plane of the polarizer were made parallel to the vertical crosshair, but the plane of obliquity was kept parallel to

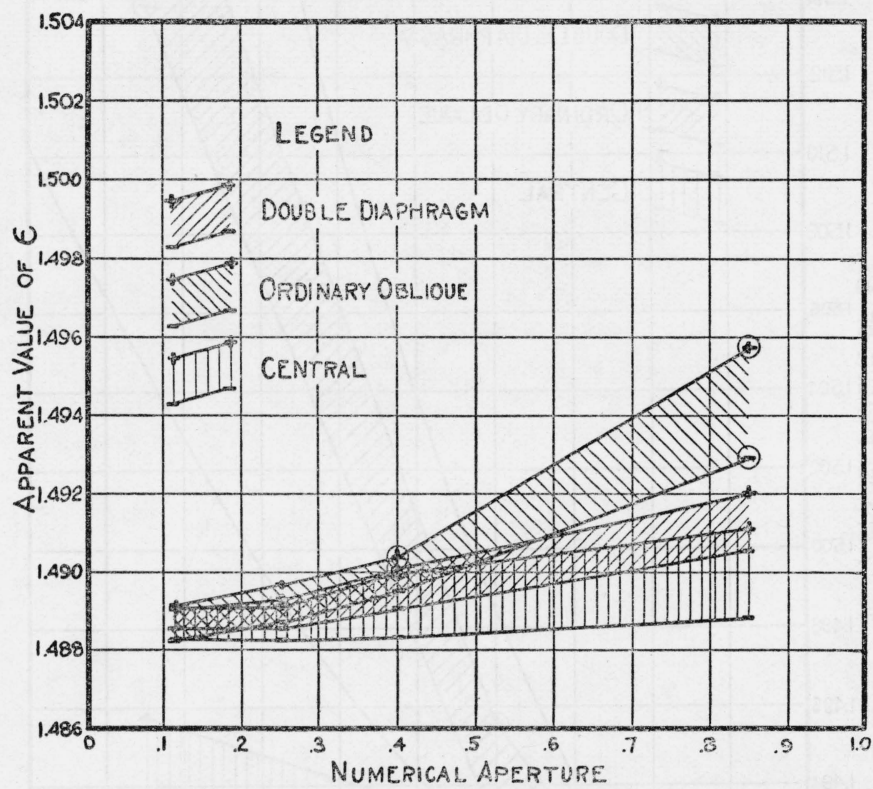

FIGURE 8.-Det \$mination of $\epsilon$, mercuric cyanide, when the plane of obliquity is normal to the optic axis.

The apparent value of $\epsilon$ is plotted against the full numerical aperture of the objective. The inaccuracies that are introduced into each method by the birefringence of the crystal are shown by the amount that the average of a zone lies above 1.4887 (true $\epsilon$ as determined from the data of this figure and figure 7 ).

the horizontal crosshair. Becke lines were considered only as they occurred along the sides of crystals.

In securing the data of figures 7 and 8 , there were instances in which the index of the liquid was indicated simultaneously to be both above and below that of the crystal. The influence of differently oblique rays produced the contradictory results. In these cases, the refractive index of the immersion liquid was carried to a value that caused one of these effects clearly to predominate, though the other effect might still be marked. Such points in the figures have been indicated by circles drawn around them.

Besides the experimental points, two theoretical curves have been drawn in figure 7 to represent apparent values of $\epsilon$ by ordinary oblique illumination. In the vertical elliptical principal section, figure 9, through the index ellipsoid of mercuric cyanide, the horizontal half- 
axis is proportional to $\epsilon$, since the unique axis of the crystal lies in the plane of the microscope stage, and the axis perpendicular to $\epsilon$ is proportional to $\omega$. The axes $\epsilon$ and $\omega$ correspond to directions of vibration and not of propagation. The ray travelling in the plane of this section and vibrating along $\epsilon_{1}$, would travel in an isotropic medium along a path making an angle $A$ with the vertical. The length of the line $\epsilon_{1}$, is proportional to the index of refraction for such a ray.

The equation of the elliptical section is

$$
\begin{gathered}
\frac{x^{2}}{\epsilon^{2}}+\frac{y^{2}}{\omega^{2}}=1, \text { but } \\
\epsilon_{1}^{2}=x^{2}+y^{2} \text { and } \\
\sin A=\frac{y}{\epsilon_{1}}, y=\epsilon_{1} \sin A .
\end{gathered}
$$

By refraction at the surface of a preparation, $\alpha$, the obliquity of the ray in air becomes such that

$$
\begin{aligned}
\sin \alpha & =\epsilon_{1} \sin A, \text { wherefore } \\
y & =\sin \alpha \\
y^{2} & =\sin ^{2} \alpha, \text { and } \\
x^{2} & =\epsilon_{1}^{2}-\sin ^{2} \alpha .
\end{aligned}
$$

Substituting these values of $x^{2}$ and $y^{2}$ in the equation of the ellipse and rearranging, we acquire the relation

$$
\sin ^{2} \alpha=\frac{\epsilon_{1}^{2} \omega^{2}-\epsilon^{2} \omega^{2}}{\omega^{2}-\epsilon^{2}}
$$

in which, using ordinary oblique illumination, $\epsilon_{1}$ is the extraordinary index of refraction for the ray, a, figure 10, which just enters or fails to enter the equatorial belt of an objective, the numerical aperture of which is $\sin \alpha$. When the diaphragm producing oblique illumination is so set that $4 / 5$ of the diameter of the objective is blocked, $4 / 10$ of the radius is open or $6 / 10$ is closed. For the rays which just succeed or fail in entering the objective at the points $b$ and $b^{1}, \sin \alpha=0.6$ N.A. Wherefore we have the two relations

$$
\begin{aligned}
& N . A=\sqrt{\frac{\epsilon_{1}^{2} \omega^{2}-\epsilon^{2} \omega^{2}}{\omega^{2}-\epsilon^{2}}} \text {, and } \\
& N . A .=\sqrt{\frac{\epsilon_{1}^{2} \omega^{2}-\epsilon^{2} \omega^{2}}{\omega^{2}-\epsilon^{2}}} \times \frac{1}{.6},
\end{aligned}
$$

which were used to plot the smooth curves in figure 7 .

The zone between the two curves corresponds to results which would simultaneously be indicated by different parts of the objective aperture if the method of matching were absolutely sensitive. 
From the figures 7 and 8 it is readily apparent that errors can become excessively large. Ordinary oblique illumination and central illumination produce larger errors when the plane of obliquity is parallel

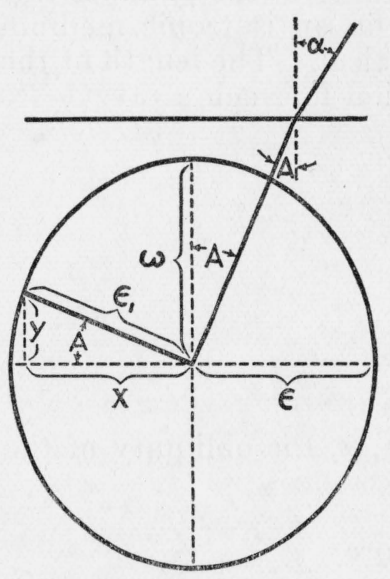

Figure 9. to the vibration direction. The double-diaphragm method produces larger errors when the plane of obliquity is normal to the vibration direction. Errors can be kept small by an intelligent use of either the Beckeline method or the double-diaphragm method. Errors become greatest when the plane of the polarizer is parallel to the horizontal crosshair, a setting which has been recommended as producing the brightest images with daylight, and the ordinary method of oblique illumination is applied by swinging the mirror to one side or shading one side of the condenser with the finger.

A uniaxial crystal with its unique axis parallel to the microscope stage was used in the foregoing tests because the errors in determining an index could be easily analyzed and interpreted. If a uniaxial crystal otherwise oriented or a biaxial crystal had been chosen instead, interpretation would have been made more difficult by the more complex dependence of the apparent index upon the azimuth as well as the inclination of the many rays in the cone of illumination. But the inferences to be drawn from such tests would have been the same and the same recommendations of procedure would have resulted.

\section{CONCLUSIONS}

The ordinary method of oblique illumination should never be employed unless, as in preliminary trials, inexact results will suffice.

All the methods are capable of greater accuracy and higher sensitivity when employed with objectives of low numerical aperture.

The double-diaphragm method has in general certain small advantages of accuracy and sensitivity over the Becke-line method. With the former, the plane of vibration of the light vector should always be parallel to the horizontal crosshair. With the latter, it should always be parallel to the vertical crosshair when those

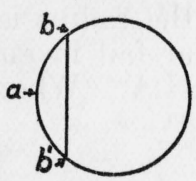

Figure 10. Becke-line effects are considered which occur along the right and left sides of the particle.

Washington, July 23, 1935. 